\title{
Dynamic analysis of thick plates including deep beams on elastic foundations using modified Vlasov model
}

\author{
Korhan Ozgan \\ Department of Civil Engineering, Faculty of Engineering, Karadeniz Technical University, Turkey \\ Tel.: +90 462377 2652; Fax: +90 462377 2606; E-mail: korhanozgan@yahoo.com \\ Received 14 April 2011 \\ Revised 25 October 2011
}

\begin{abstract}
Dynamic analysis of foundation plate-beam systems with transverse shear deformation is presented using modified Vlasov foundation model. Finite element formulation of the problem is derived by using an 8-node (PBQ8) finite element based on Mindlin plate theory for the plate and a 2-node Hughes element based on Timoshenko beam theory for the beam. Selective reduced integration technique is used to avoid shear locking problem for the evaluation of the stiffness matrices for both the elements. The effect of beam thickness, the aspect ratio of the plate and subsoil depth on the response of plate-beam-soil system is analyzed. Numerical examples show that the displacement, bending moments and shear forces are changed significantly by adding the beams.
\end{abstract}

Keywords: Finite element method, thick plate, deep beam, elastic foundation, Vlasov model, shear locking problem, dynamic analysis

\section{Introduction}

Plates on elastic foundation are used in construction of mat and raft foundations, highways and airfield pavements and they are subjected to dynamic loads normal to their plane. So it is important for engineers to know the dynamic behavior of the plates on elastic foundations before structural designs. Kirchhoff plate and Euler-Bernoulli beam theories are commonly used for the analysis but the effects of transverse shear deformation are neglected in both the theories. However the effects of the shear deformation become more important as the structural element gets thicker. Mindlin plate theory and Timoshenko beam theory include the effects of transverse shear deformation. However one can face with the shear locking problem when these theories are used for the elements with small thicknesses. Reduced or selective reduced integration techniques are recommended to avoid this problem. On the other hand the subsoil is usually represented by spring elements in the soil-structure interaction problems. The springs are assumed to be discrete in Winkler model and any interaction between the springs is ignored. However there is not only pressure but also bending moments or rotations at the point of contact between structure and foundation. Several researchers tried to improve the Winkler Model by considering a second parameter for the interaction of the springs. But the disadvantage of two parameter model is the necessity to determine the values of subgrade reaction (or Winkler moduli, $k$ ) and shear parameter (or Pasternak moduli, 2t) arbitrarily. So Vlasov and Leont'ev introduced another parameter $(\gamma)$ to compute Winkler and Pasternak moduli from the material properties of the soil. But the authors did not provide any mechanism for the calculation of the third parameter $(\gamma)$. Vallabhan and Das developed an iterative method for the calculation of the $\gamma$ parameter and called their model as Modified Vlasov Model. In this study Modified Vlasov Model is used for the dynamic analysis of thick plates including deep beams 


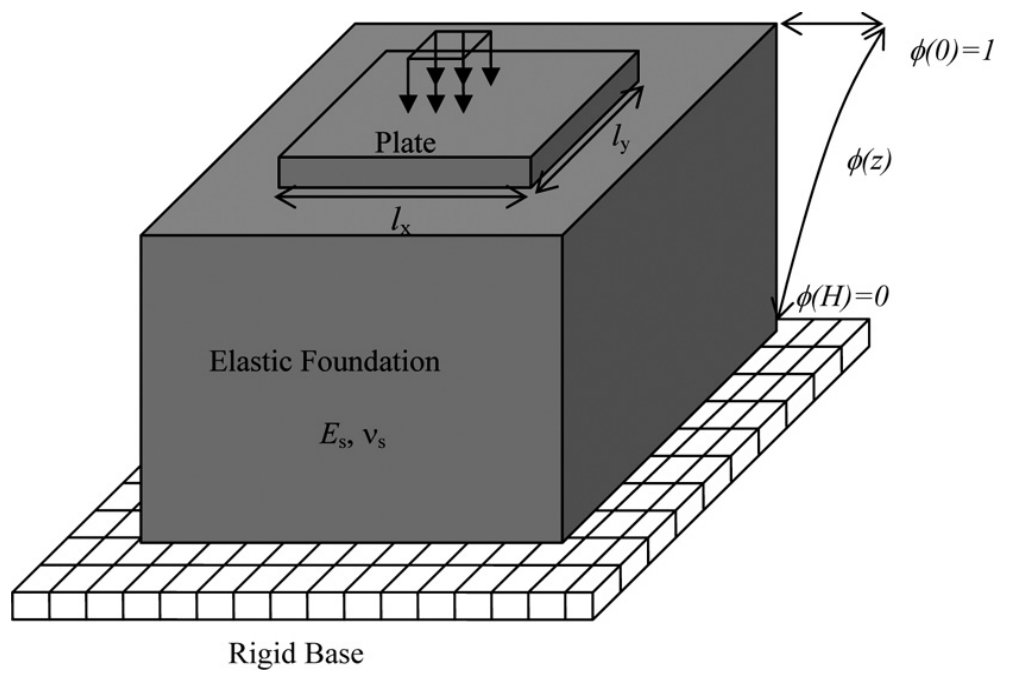

Fig. 1. A plate resting on an elastic foundation.

on elastic foundation incorporating shear deformation effects in the structures and the shear interaction effect within the soil [1].

Many studies concerning dynamic analysis of plates on elastic foundation are performed. Labuschange et al. [2] analyzed a plate-beam system in which Reissner-Mindlin plate model is combined with the Timoshenko beam model, and calculated natural frequencies and vibration modes for the system. Sapountzakis and Katsikadelis [3] presented an optimized model for the analysis of plates reinforced with beams. Shen et al. [4] performed the free and forced vibration analysis of Reissner-Mindlin plates resting on Pasternak-type elastic foundation. Sapountzakis and Mokos [5] presented a general solution for the dynamic analysis of plates stiffened by arbitrarily placed parallel beams of arbitrary doubly symmetric cross section subjected to arbitrary dynamic loading. Gorman [6] obtained analytical type solutions for the free vibration frequencies and mode shapes of thin corner supported rectangular plates with symmetrically distributed reinforcing beams. Van Rensburg et al. [7] studied on vibration of platebeam system consisting of a Reissner-Mindlin plate and a Timoshenko beam. Yu et al. [8] presented the dynamic response of Reissner-Mindlin plate resting on an elastic foundation of the Winkler-type and Pasternak-type using an analytical-numerical method. Wen and Aliabadi [9] used boundary element method for the analysis of Mindlin plates on elastic foundation subjected to dynamic load.

Ozgan and Daloglu [10] studied the effects of transverse shear strain on plates resting on elastic foundation. They compared 4 node and 8 node Mindlin plate element with both of full (FI) and selective reduced integration (SRI) technique in the study and the results showed that 8-node element (SRI) was more reliable than 8 node (FI), 4-node (FI) and 4-node (SRI) elements for analyzing thin and thick plates on elastic foundation under any type of load cases and boundary conditions.

In this paper; 8-node (PBQ8) Mindlin plate elements and 2-node Hughes beam element based on Timoshenko beam theory were adopted since the shear strains are included for the dynamic analysis of thick plates resting on elastic foundation. For this purpose a computer program was coded in Visual Fortran 6.6. Newmark- $\beta$ method was used for time integration. The selective reduced integration technique was used to obtain stiffness matrices to avoid shear locking problem. A verification study is performed first by comparing the results with those given in the literature. Later the study is expanded to investigate the effect of the beam thickness, the aspect ratio of the plate and the subsoil depth on the behavior of the structure soil system.

\section{Modified Vlasov model}

For a plate on elastic foundation as shown in Fig. 1, the lateral displacements $(\bar{u}$ and $\bar{v})$ in the soil are negligible compared to the vertical displacement $(\bar{w})$ in the $z$ direction, and, hence, it is assumed that 


$$
\begin{aligned}
& \bar{u}(x, y, z)=0 \\
& \bar{v}(x, y, z)=0
\end{aligned}
$$

and

$$
\bar{w}(x, y, z)=w(x, y) \phi(z)
$$

where $\phi(z)$ defines the variation of the deflection $\bar{w}(x, y, z)$ in the $z$ direction. This function depicted in Fig. 1, and since $w(x, y)$ is displacement of the plate-beam system in the $z$ direction, $\phi(0)$ is equal to 1 , and $\phi(H)$ is equal to 0 (zero).

Total potential energy of the plate-soil system can be written as

$$
\begin{aligned}
\prod= & \frac{1}{2} \int_{\Omega}\left(\frac{\partial^{2} w}{\partial x^{2}}, \frac{\partial^{2} w}{\partial y^{2}}, 2 \frac{\partial^{2} w}{\partial x \partial y}\right)[D]\left(\frac{\partial^{2} w}{\partial x^{2}}, \frac{\partial^{2} w}{\partial y^{2}}, 2 \frac{\partial^{2} w}{\partial x \partial y}\right)^{T} d x d y \\
& +\frac{1}{2} \int_{-\infty}^{+\infty} \int_{-\infty}^{+\infty}\left\{k w^{2}+2 t(\nabla w)^{2}\right\} d x d y-\int_{\Omega} q w d x d y
\end{aligned}
$$

where $[D]$ and $q$ are the flexural rigidity of the plate and load on plate respectively. $k$ and $2 t$ in above expression are the soil parameters and may be defined as

$$
\begin{aligned}
k & =\int_{0}^{H} \bar{E}\left(\frac{\partial \phi}{\partial z}\right)^{2} d z \\
2 t & =\int_{0}^{H} G \phi^{2} d z
\end{aligned}
$$

where $\bar{E}$ is the oedometer modulus and calculated by the equation of $\bar{E}=E_{s}\left(1-\nu_{s}\right) /\left[\left(1+\nu_{s}\right)\left(1-2 \nu_{s}\right)\right], H$ is the subsoil depth, $E_{s}$ is modulus of elasticity of the subsoil, $\nu_{s}$ is the Poisson ratio of the subsoil and $G$ is the shear modulus.

The equilibrium equation of the plate on an elastic foundation can be written as follows

$$
D \nabla^{4} w-2 t \nabla^{2} w+k w=q
$$

where $\nabla^{2}$ is the Laplace and $\nabla^{4}$ is the biharmonic operators. $\phi(z)$ can be expressed as

$$
\phi(z)=\frac{\sinh \left(\gamma\left(1-\frac{z}{H}\right)\right)}{\sinh \gamma}
$$

where $\gamma$ represents the vertical deformation parameter within the subsoil and is calculated using the equation shown below.

$$
\left(\frac{\gamma}{H}\right)^{2}=\frac{1-2 \nu_{s}}{2\left(1-\nu_{s}\right)} \frac{\int_{-\infty}^{+\infty} \int_{-\infty}^{+\infty}(\nabla w)^{2} d x d y}{\int_{-\infty}^{+\infty} \int_{-\infty}^{+\infty} w^{2} d x d y}
$$

As seen from the Eqs (5) and (8) the modulus of subgrade reaction, $k$, and the second parameter $2 t$ representing the shear deformation of the soil, are both dependent on the vertical deformation function $\phi$ and the depth of the soil $H$ whereas the value of $\gamma$ varies with the displacement of the plate and the depth of the subsoil. The parameter $\gamma$ can be evaluated after determining $w(x, y)$ which satisfies differential equation below and around of the plate. The solution technique for $\gamma$ parameter is an iterative process. More details for the modified Vlasov model can be found in references [11-13]. 


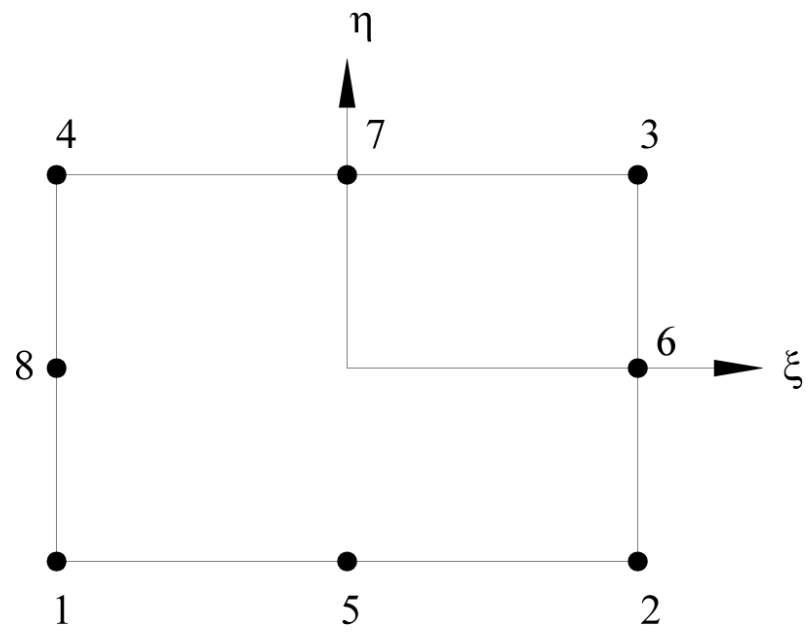

Fig. 2. 8-node Mindlin plate element.

\section{Finite element modelling}

The general equation of motion for the plate-beam-soil system is given by

$$
[M]\{\ddot{w}\}+[K]\{w\}=\{F\}
$$

where $[K]$ is the stiffness matrix of the plate-beam-soil system, $[M]$ is the mass matrix of the plate-beam-soil system, $\{F\}$ is the applied load vector, $w$ and $\ddot{w}$ are the displacement and acceleration vector of the plate, respectively. In this study the Newmark- $\beta$ method is used for the time integration of Eq. (9) by using the average acceleration method. Evaluation of the stiffness and mass matrices are given in the following sections for a plate including beams resting on an elastic foundation.

\subsection{Evaluation of the stiffness matrix}

An 8-node (PBQ8) rectangular finite element based on Mindlin theory are used to develop the element stiffness matrices (Fig. 2).

The displacements at each node are

$$
w, \varphi_{x}, \varphi_{y}
$$

where $w$ is the transverse displacement, $\varphi_{x}, \varphi_{y}$ are the rotations of the normal to the undeformed middle surface. It is assumed that $w, \varphi_{x}$ and $\varphi_{y}$ vary quadratically for PBQ8 over the element so that

$$
\begin{aligned}
& u=z \varphi_{y}=z \sum_{i=1}^{8} N_{i} \varphi_{y i} \\
& v=-z \varphi_{x}=-z \sum_{i=1}^{8} N_{i} \varphi_{x i} \\
& w=\sum_{i=1}^{8} N_{i} w_{i}
\end{aligned}
$$

The displacement functions for PBQ8 elements are given as 


$$
\left[\begin{array}{c}
w \\
\varphi_{x} \\
\varphi_{y}
\end{array}\right]=\left[\begin{array}{cccccccccc}
N_{1} & 0 & 0 & N_{2} & 0 & 0 & \ldots & N_{8} & 0 & 0 \\
0 & N_{1} & 0 & 0 & N_{2} & 0 & \ldots & 0 & N_{8} & 0 \\
0 & 0 & N_{1} & 0 & 0 & N_{2} & \ldots & 0 & 0 & N_{8}
\end{array}\right]\left[\begin{array}{c}
w_{1} \\
\varphi_{x 1} \\
\varphi_{y 1} \\
w_{2} \\
\varphi_{x 2} \\
\varphi_{y 2} \\
\cdot \\
\cdot \\
\cdot \\
w_{8} \\
\varphi_{x 8} \\
\varphi_{y 8}
\end{array}\right]
$$

in which $N_{1}, N_{2}, N_{3}, N_{4}, N_{5}, N_{6}, N_{7}$ and $N_{8}$ are the shape functions as given in [14,15].

The stiffness matrices of the plate-soil system can be evaluated as

$$
U=\frac{1}{2}\left\{w_{e}\right\}^{t}\left(\left[k_{p}\right]+\left[k_{w}\right]+\left[k_{2 t}\right]\right)\left\{w_{e}\right\}
$$

where $\left[k_{p}\right],\left[k_{w}\right]$ and $\left[k_{2 t}\right]$ are stiffness matrix of the plate, vertical deflection element stiffness matrix of the foundation and shear deformation element stiffness matrix of the foundation, respectively. $\left\{w_{e}\right\}$ is the nodal displacement vector for an element containing 24 components.

The stiffness matrices for the beam elements have to be added to total system matrix in order to consider plate with beams. The displacement function of Hughes element (Fig. 3) based on Timoshenko beam theory can be expressed as

$$
\left[\begin{array}{l}
w \\
\varphi
\end{array}\right]=\left[\begin{array}{cccc}
N_{1} & 0 & N_{2} & 0 \\
& N_{1} & & N_{2}
\end{array}\right]\left[\begin{array}{l}
w_{1} \\
\varphi_{1} \\
w_{2} \\
\varphi_{2}
\end{array}\right]
$$

where $N_{1}$ and $N_{2}$ are the shape functions of the element, $w$ is the transverse displacement and $\phi$ is the rotation at the ends of the beam element. Calculation of the moment inertia of the beam, $I$, has to be done carefully when a plate and a beam element work together, as indicated in Fig. 4. if depth of the beam is $t_{b}$ and depth of the plate is $t_{p}$, the distance from the middle surface of the slab to centroidal axis of the beam, $r$, is

$$
r=\frac{1}{2}\left(t_{p}+t_{b}\right)
$$

In this study, the selective reduced integration rule on the shear terms is used to obtain the element stiffness matrix of the plate $\left[k_{p}\right]$ to avoid shear locking problem under the thin plate limit. The number of points of integration for shear energy terms of stiffness matrix is reduced one step for both of the beam and the plate element. The element stiffness matrices are given in explicit forms by Ozgan [13] for the plate element and by Ozgan and Daloglu [10] for the vertical deflection and the shear deformation of the foundation.

Boundary conditions need to be applied before solving the system of equations. The effect of the infinite soil domain outside the plate is applied as equivalent stiffness parameters on the plate boundaries in the modified Vlasov model. Equivalent forces due to surrounding soil domain on the boundary of the plate are computed as a function of the displacement on the boundary $[10,12]$.

\subsection{Evaluation of the mass matrix}

The total kinetic energy of the structure-soil system may be written as

$$
\pi_{k}=\frac{1}{2} \int_{\Omega}\{\dot{w}\}^{T}[\mu]\{\dot{w}\} d \Omega
$$




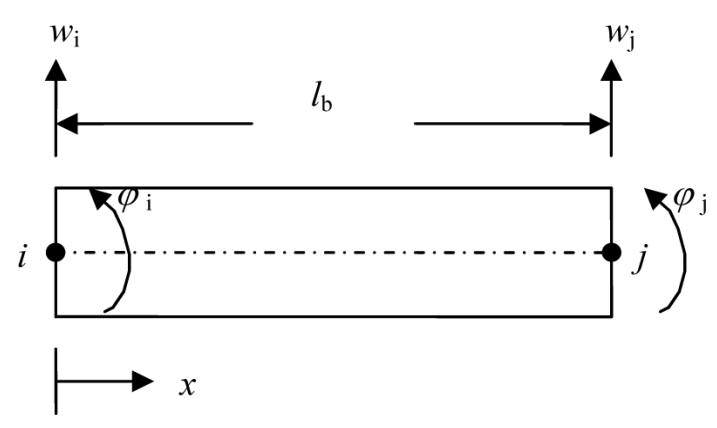

Fig. 3. Timoshenko beam element.

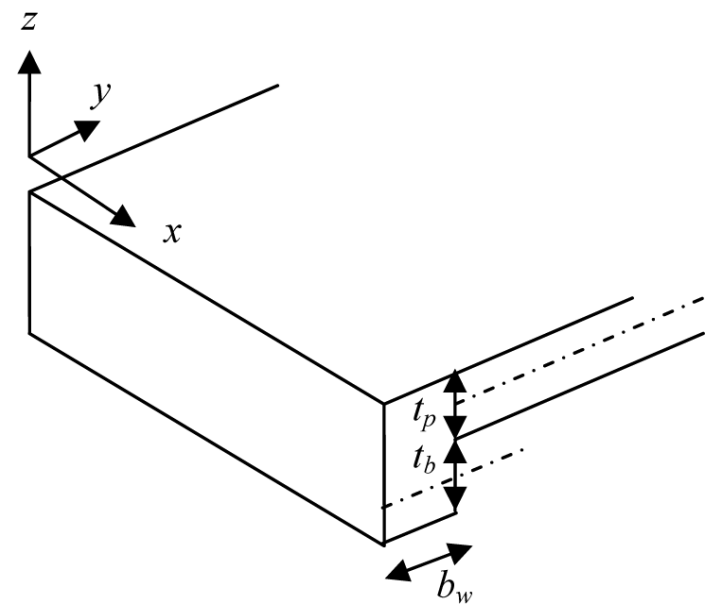

Fig. 4. A plate with beam.

where $[\mu]$ is the mass density matrix and $\{\dot{w}\}$ represents the partial derivative of the vector of generalized displacement with respect to time variable. The general formula for the consistent mass matrix, $[M]$, can be written by substituting $w=\left[N_{1}\right]\left\{w_{e}\right\}$ into Eq. (16)

$$
[M]=\int_{\Omega}\left\{N_{1}\right\}^{T}[\mu]\left\{N_{1}\right\} d \Omega
$$

The matrix $[\mu]$ for plate in Eq. (17) is a square symmetric matrix of the form

$$
[\mu]=\left[\begin{array}{ccc}
\rho_{p} t_{p}+\frac{1}{3} \rho_{s} H & 0 & 0 \\
0 & \frac{1}{12} \rho_{p} t_{p}^{3} & 0 \\
0 & 0 & \frac{1}{12} \rho_{p} t_{p}^{3}
\end{array}\right]
$$

where $\rho_{p}$ is the plate density, $t_{p}$ is the plate thickness and $\rho_{s}$ is the mass density of the soil. The matrix form of $[\mu]$ for beam element is

$$
[\mu]=\left[\begin{array}{cc}
\rho_{b} t_{b}+\frac{1}{3} \rho_{s} H & 0 \\
0 & \frac{1}{12} \rho_{b} t_{b}^{3}
\end{array}\right]
$$

The term of $\rho_{s} H / 3$ shows the effect of mass density of the subsoil and it is calculated by substituting

$$
\phi(z)=1-z / H \text { into } \int_{0}^{H} \rho_{s} \phi^{2}(z),[16] .
$$

The mass matrices for the beam elements have to be added to total system matrix in order to consider plate with beams as mentioned in the calculation of stiffness matrix. The matrices for PBQ8 plate element and 2-node beam element are presented in Appendixs A and B respectively.

\section{Numerical examples}

The dynamic analysis of a uniformly distributed loaded plate on elastic foundation is performed and results are compared with those of Daloglu et al. [17] first. Then the same example is solved by including beams in both the directions as shown in Fig. 5. Later a parametric study is carried out for various values of subsoil depth $(H)$, aspect ratios $\left(l_{y} / l_{x}\right)$ and the thickness of beam. Time histories of maximum displacements are given for only some cases because presentation of all of the time histories of the displacements at different points on the plate would take up excessive space. The effects of subsoil depth $(H)$, aspect ratios $\left(l_{y} / l_{x}\right)$ and the thickness of beam on maximum displacements, bending moments and shear forces of the plates are presented in graphical and tabular form. 
Table 1

Properties of plate-soil system used in this study

\begin{tabular}{ll}
\hline Parameters & Quantity \\
\hline Elasticity modulus of the plate, $E_{p}$ & $27000 \mathrm{MPa}$ \\
Poisson ratio of the plate, $\nu_{p}$ & 0.20 \\
Thickness of the plate, $t_{p}$ & $0.5 \mathrm{~m}$ \\
Elasticity modulus of the soil, $E_{s}$ & $20 \mathrm{MPa}$ \\
Poisson ratio of the soil, $\nu_{s}$ & 0.25 \\
Mass densities of the plate, $\rho_{p}$ & $2500 \mathrm{~kg} / \mathrm{m}^{3}$ \\
Mass densities of the soil, $\rho_{s}$ & $1700 \mathrm{~kg} / \mathrm{m}^{3}$ \\
Distributed load, $Q$ & $30 \mathrm{kPa}$ \\
Shorter edge length of the plate, $l_{x}$ & $10 \mathrm{~m}$ \\
Aspect ratio of the plate, $l_{y} / l_{x}$ & $1.0,1.5$ and 2.0 \\
Subsoil depth, $H$ & 5,10 and $15 \mathrm{~m}$ \\
Beam sections $\left(\mathrm{b}_{w} \times t_{b}\right)$ & without beam, $1.00 \mathrm{~m} \times 1.00 \mathrm{~m}$ and $1.0 \mathrm{~m} \times 1.5 \mathrm{~m}$ \\
\hline
\end{tabular}

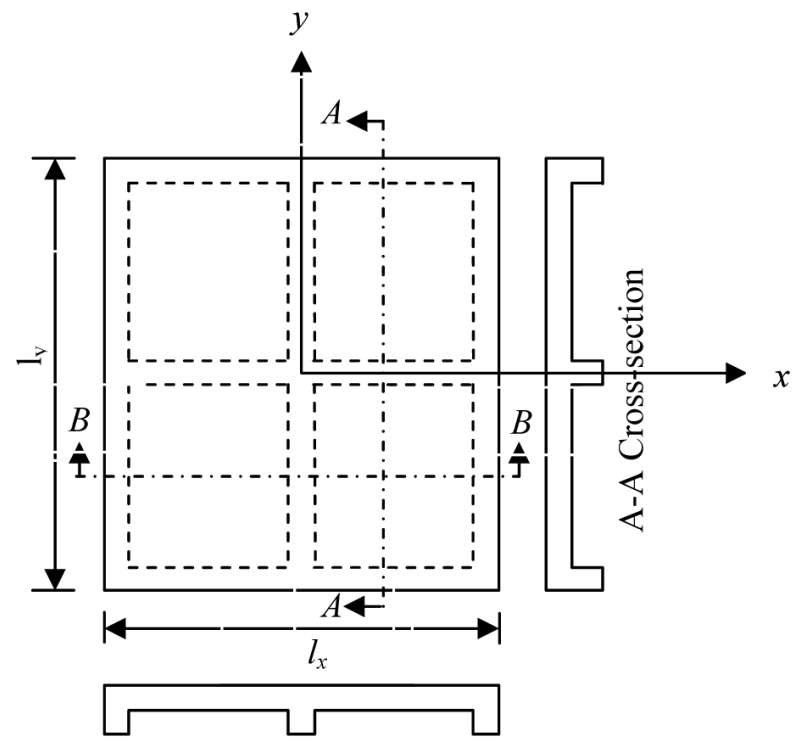

B-B Cross-section

Fig. 5. Illustration of plate with beams.

The properties of the plate-soil system are as follows (Table 1). The modulus of elasticity of the plate is $E_{p}=$ $27000 \mathrm{MPa}$, poisson ratio of the plate is $\nu_{p}=0.20$, thickness of the plate is $0.5 \mathrm{~m}$, the modulus of elasticity of the subsoil is $E_{s}=20 \mathrm{MPa}$, poisson ratio of the plate is $\nu_{s}=0.25$. The mass densities of the plate and subsoil are taken to be $\rho_{p}=2500 \mathrm{~kg} / \mathrm{m}^{3}$ and $\rho_{s}=1700 \mathrm{~kg} / \mathrm{m}^{3}$ respectively. The uniformly distributed load on the plate is $30 \mathrm{kPa}$. The plate dimensions are $10 \mathrm{~m} \times 10 \mathrm{~m}$. The analysis is performed for three different subsoil depth, $\mathrm{H}=5,10$ and $15 \mathrm{~m}$ and the aspect ratio $\left(l_{y} / l_{x}\right)$ is considered as 1.0, 1.5 and 2.0 keeping $l_{x}$ constant at $10 \mathrm{~m}$ for each subsoil depth. The example is first solved for a plate without beam. Then, the analysis repeated for $1 \mathrm{~m}$ and $1.5 \mathrm{~m}$ thickness of the beam while the width of the beam is kept constant at $1 \mathrm{~m}$. The beams are placed on the edges and on centerlines of the plate in each direction (Fig. 5).

A convergence study for the mesh size and the time increment is performed first for the sake of accuracy. It is decided that the results are accurate enough when equally spaced 6 elements for $10 \mathrm{~m}$ length plate and 12 elements for $10 \mathrm{~m}$ length beam are used with a $0.01 \mathrm{~s}$ time increment.

The maximum displacements for $l_{y} / l_{x}=1.0$ and $2.0, h=0.5 \mathrm{~m}$ and $H=5 \mathrm{~m}$ are compared first with the results obtained in reference [17] to verify the accuracy of the present formulation and presented in Table 2 . $t$ in Table 2 stands for the time in which the center displacement of the plate reaches its absolute maximum value. Daloglu et al. [17] solved the problem using MZC rectangular element, which is originally developed by Melosh, Zienkiewicz 
Table 2

Comparison of maximum displacement of plate on elastic foundation

\begin{tabular}{|c|c|c|c|c|}
\hline \multirow{3}{*}{ Finite Element } & \multicolumn{4}{|c|}{ Distributed load } \\
\hline & \multicolumn{2}{|c|}{$10 \mathrm{~m} \times 10 \mathrm{~m}$ plate } & \multicolumn{2}{|c|}{$10 \mathrm{~m} \times 20 \mathrm{~m}$ plate } \\
\hline & $t$ time $(\mathrm{s})$ & $\mathrm{w}_{\max }(\mathrm{mm})$ & $t$ time $(\mathrm{s})$ & $\mathrm{w}_{\max }(\mathrm{mm})$ \\
\hline Ref. [17] & 3.080 & 10.200 & 2.010 & 12.800 \\
\hline PBQ8(SRI) & 7.590 & 10.170 & 5.230 & 12.840 \\
\hline
\end{tabular}

Table 3

Maximum displacements and bending moments of plates on elastic foundation

\begin{tabular}{|c|c|c|c|c|c|c|}
\hline \multirow{2}{*}{$H(m)$} & \multirow{2}{*}{$l_{x} / l_{y}$} & \multirow{2}{*}{ Beam Section } & \multicolumn{4}{|c|}{ Distributed load } \\
\hline & & & time $(\mathrm{s})$ & $\mathrm{w}_{\max }(\mathrm{mm})$ & $\mathrm{M}_{x}(\mathrm{kNm})$ & $\mathrm{M}_{y}(\mathrm{kNm})$ \\
\hline \multirow{9}{*}{5} & \multirow{3}{*}{1.0} & without beam & 7.59 & 10.170 & 110.29 & 110.29 \\
\hline & & $1.0 \mathrm{~m} \times 1.0 \mathrm{~m}$ & 8.64 & 7.679 & 14.36 & 14.36 \\
\hline & & $1.0 \mathrm{~m} \times 1.5 \mathrm{~m}$ & 3.36 & 7.443 & 5.91 & 5.91 \\
\hline & \multirow{3}{*}{1.5} & without beam & 2.44 & 11.841 & 113.82 & 102.67 \\
\hline & & $1.0 \mathrm{~m} \times 1.0 \mathrm{~m}$ & 3.71 & 8.744 & 23.77 & 16.86 \\
\hline & & $1.0 \mathrm{~m} \times 1.5 \mathrm{~m}$ & 3.32 & 8.234 & 9.78 & 6.88 \\
\hline & \multirow{3}{*}{2.0} & without beam & 5.23 & 12.840 & 123.45 & 88.61 \\
\hline & & $1.0 \mathrm{~m} \times 1.0 \mathrm{~m}$ & 5.67 & 9.659 & 29.39 & 16.70 \\
\hline & & $1.0 \mathrm{~m} \times 1.5 \mathrm{~m}$ & 3.70 & 8.853 & 13.31 & 6.67 \\
\hline \multirow{10}{*}{10} & \multirow{3}{*}{1.0} & without beam & 7.03 & 14.002 & 132.50 & 132.50 \\
\hline & & $1.0 \mathrm{~m} \times 1.0 \mathrm{~m}$ & 0.98 & 10.506 & 20.25 & 20.25 \\
\hline & & $1.0 \mathrm{~m} \times 1.5 \mathrm{~m}$ & 8.27 & 10.111 & 8.12 & 8.12 \\
\hline & \multirow{3}{*}{1.5} & without beam & 9.79 & 16.999 & 140.49 & 120.97 \\
\hline & & $1.0 \mathrm{~m} \times 1.0 \mathrm{~m}$ & 9.21 & 12.605 & 33.80 & 25.31 \\
\hline & & $1.0 \mathrm{~m} \times 1.5 \mathrm{~m}$ & 5.74 & 11.757 & 14.29 & 10.51 \\
\hline & \multirow{3}{*}{2.0} & without beam & 0.64 & 18.851 & 153.23 & 101.76 \\
\hline & & $1.0 \mathrm{~m} \times 1.0 \mathrm{~m}$ & 6.94 & 14.399 & 43.61 & 25.19 \\
\hline & & $1.0 \mathrm{~m} \times 1.5 \mathrm{~m}$ & 2.31 & 13.083 & 19.45 & 11.20 \\
\hline & \multirow{3}{*}{1.0} & without beam & 8.56 & 15.940 & 167.20 & 167.20 \\
\hline \multirow{8}{*}{15} & & $1.0 \mathrm{~m} \times 1.0 \mathrm{~m}$ & 0.95 & 11.709 & 22.91 & 22.91 \\
\hline & & $1.0 \mathrm{~m} \times 1.5 \mathrm{~m}$ & 4.23 & 11.228 & 9.36 & 9.36 \\
\hline & \multirow{3}{*}{1.5} & without beam & 9.65 & 19.426 & 160.41 & 129.78 \\
\hline & & $1.0 \mathrm{~m} \times 1.0 \mathrm{~m}$ & 6.65 & 14.505 & 38.35 & 29.58 \\
\hline & & $1.0 \mathrm{~m} \times 1.5 \mathrm{~m}$ & 9.58 & 13.466 & 16.49 & 12.70 \\
\hline & \multirow{3}{*}{2.0} & without beam & 7.12 & 21.980 & 167.98 & 114.50 \\
\hline & & $1.0 \mathrm{~m} \times 1.0 \mathrm{~m}$ & 5.78 & 16.897 & 49.00 & 29.32 \\
\hline & & $1.0 \mathrm{~m} \times 1.5 \mathrm{~m}$ & 2.94 & 15.318 & 22.58 & 13.75 \\
\hline
\end{tabular}

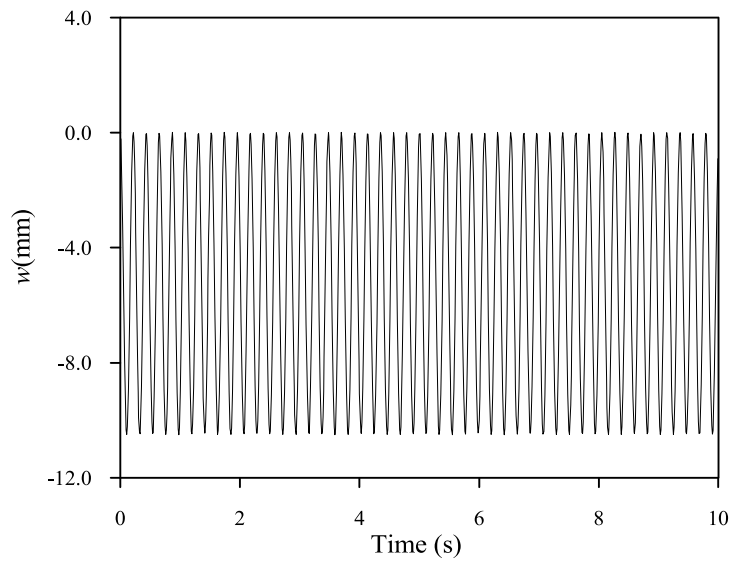

(a) $\mathrm{H}=10 \mathrm{~m}, l_{y} / l_{x}=1.0$ and $1.00 \times 1.00$ beam

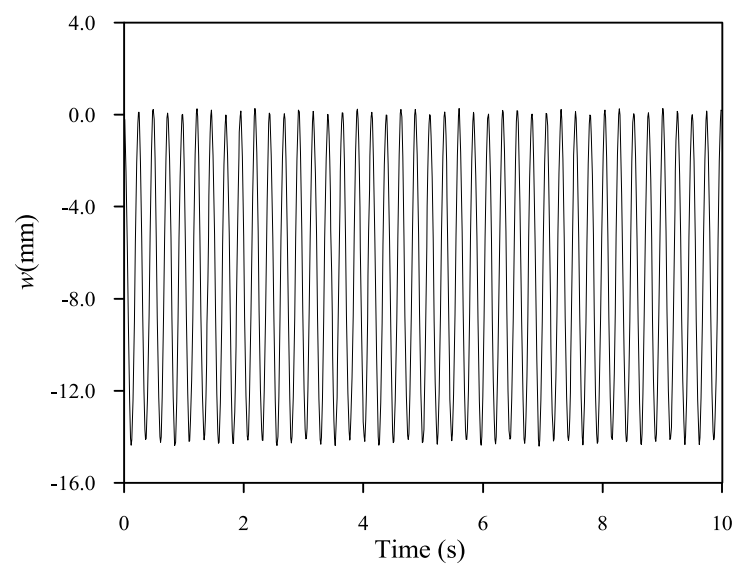

(b) $\mathrm{H}=10 \mathrm{~m}, l_{y} / l_{x}=2.0$ and $1.00 \times 1.00$ beam

Fig. 6. The time histories of the center displacements of the plate. 

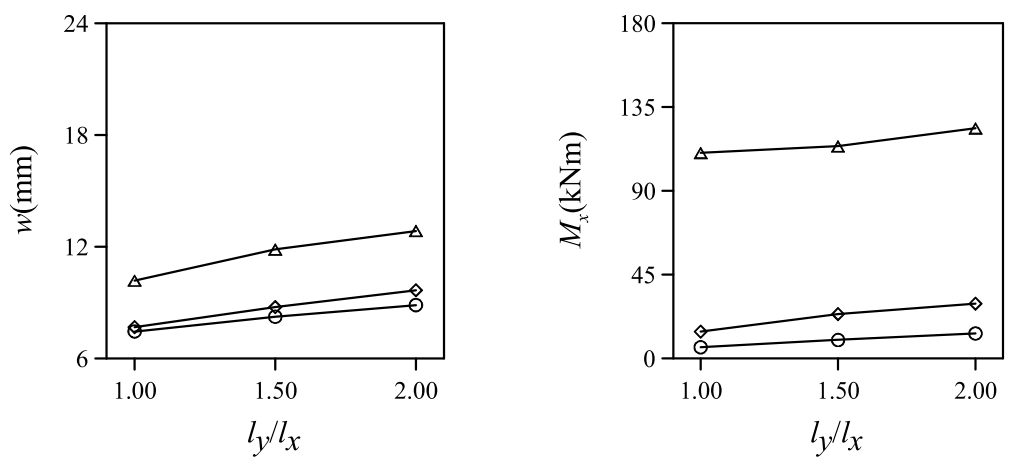

(a) $\mathrm{H}=5 \mathrm{~m}$
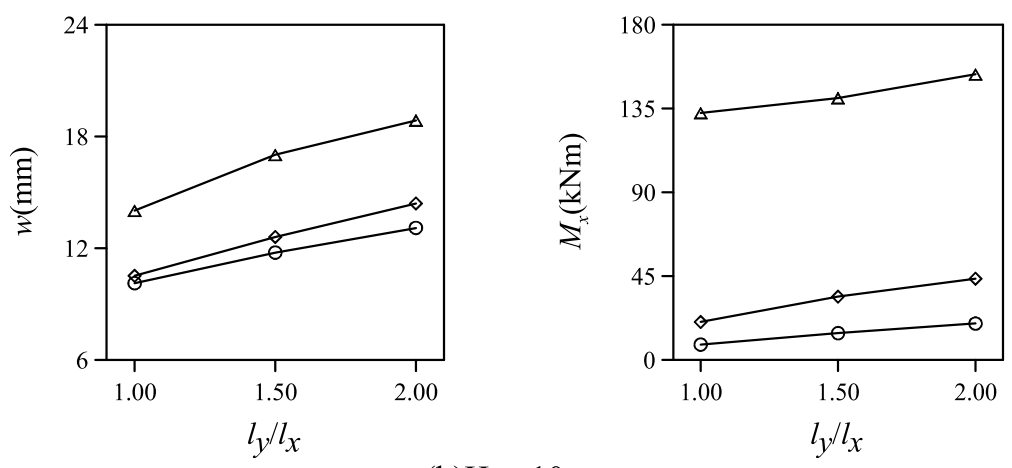

(b) $\mathrm{H}=10 \mathrm{~m}$
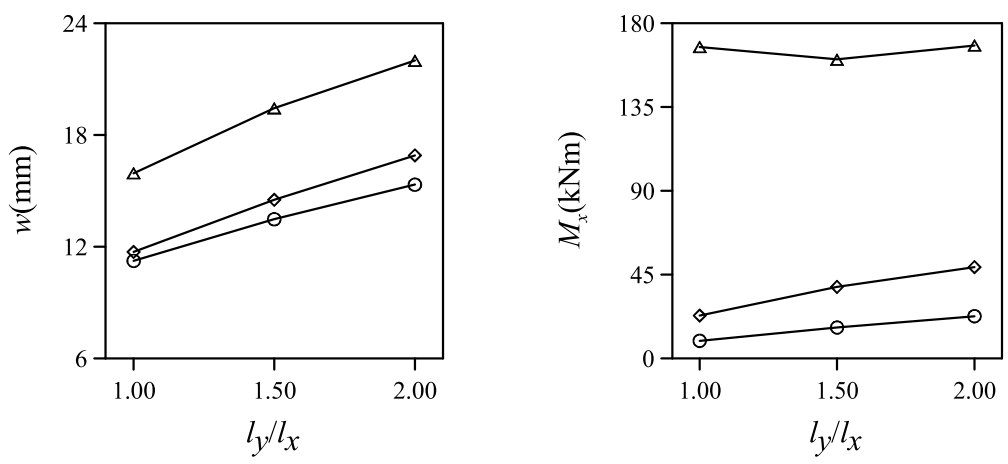

(c) $\mathrm{H}=15 \mathrm{~m}$

Fig. 7. The variation of the maximum displacement and bending moments with aspect ratio. $-\Delta-$, without beam; $-\diamond-, 1.00 \times 1.00$ beam; $-\mathrm{O}-, 1.00 \times 1.50$ beam.

and Cheung [15], based on Kirchoff plate theory in which transverse shear strains are omitted. The same example is solved by PBQ8 finite element using selective reduced integration techniques. As seen from Table 2 the results are in a good agreement with the reference results.

The results obtained for various values of aspect ratio $\left(l_{y} / l_{x}\right)$, thickness of beam and subsoil depth $(H)$ are given in Table 3. The time histories of the center displacements for $l_{y} / l_{x}=1.0$ and $2.0,1.00 \mathrm{~m} \times 1.00 \mathrm{~m}$ beam cross section and $H=10 \mathrm{~m}$ are presented in Fig. 6 . The figure indicates that the time histories of the center displacement of the plate differ from each other depending on the dynamic characteristics of the system.

The variation of the maximum displacement and corresponding bending moments of the plate with various values of aspect ratio $\left(l_{y} / l_{x}\right)$ is plotted in Fig. 7 in order to show the effects of the changes in these parameters better. The maximum displacement and bending moment increase with the aspect ratio $\left(l_{y} / l_{x}\right)$. 

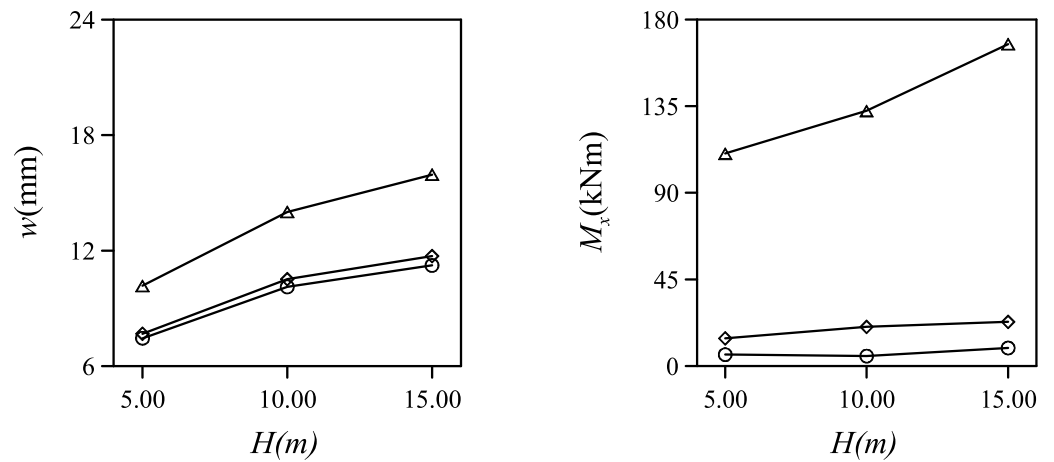

(a) $l_{y} / l_{x}=1.0$
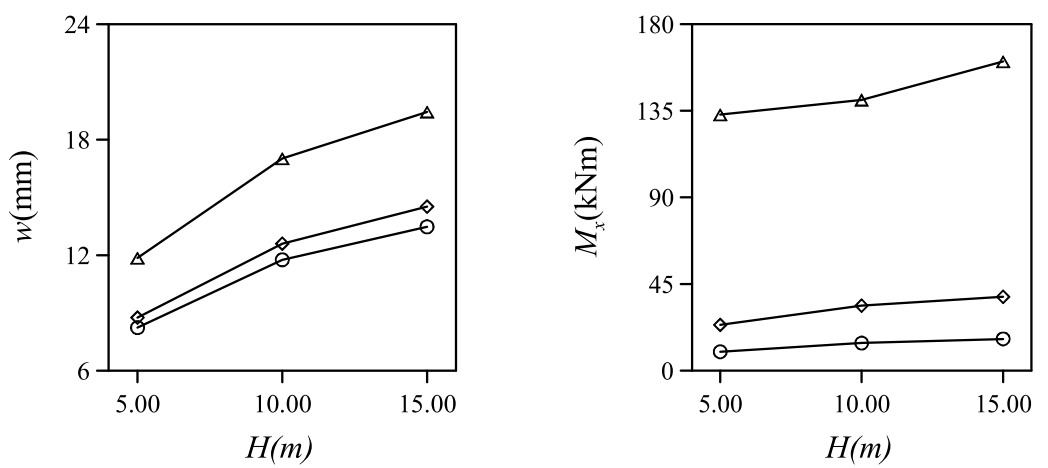

(b) $l_{y} / l_{x}=1.5$
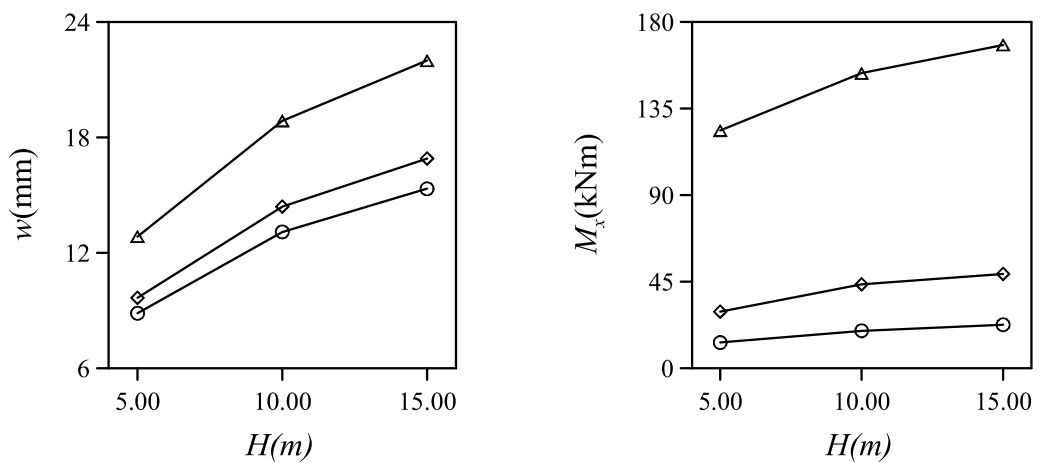

(c) $l_{y} / l_{x}=2.0$

Fig. 8. The variation of the maximum displacement and bending moments with subsoil depth. $-\Delta-$, without beam; $-\diamond-, 1.00 \times 1.00$ beam; $-\mathrm{O}-, 1.00 \times 1.50$ beam.

The variation of the maximum displacement and corresponding bending moment of the plate with various values of subsoil depth $(H)$ is plotted in Fig. 8 in order to show the effects of the changes in these parameters better. The maximum displacement and bending moment increase with increasing subsoil depth $(H)$ up to an effective depth of soil stratum. Taking deeper depth of the soil doesn't affect the result considerably but taking shallow depth may result with an unrealistic solution if there is not a relatively rigid foundation at a certain depth [18]. This behavior is understandable in that a plate on elastic foundation with larger subsoil depth becomes more flexible. The increase in the maximum displacement decreases with increasing subsoil depth $(H)$.

The variation of the maximum displacement, corresponding bending moment and shear forces throughout centerlines in the $x$ direction is plotted in Fig. 9. As can be seen from the plots, the displacement, bending moments and shear forces are changed significantly by adding the beams. The displacement, bending moment and shear forces 


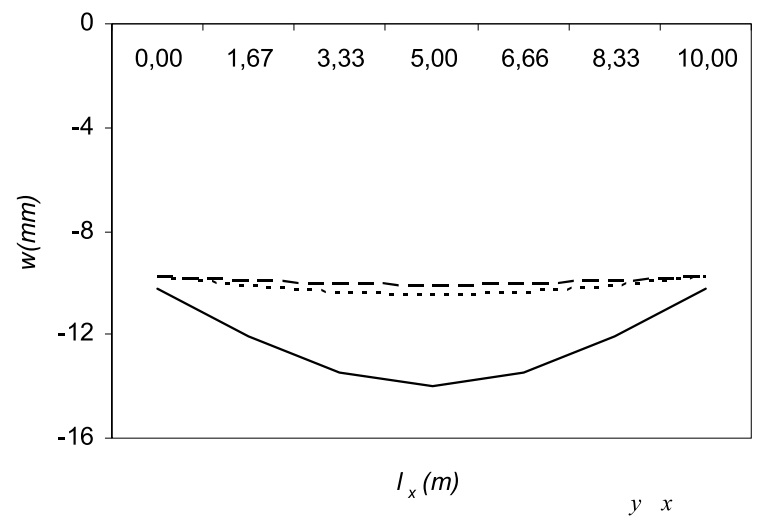

(a) Maximum displacements for $\mathrm{H}=10 \mathrm{~m}, l / l=1.0$

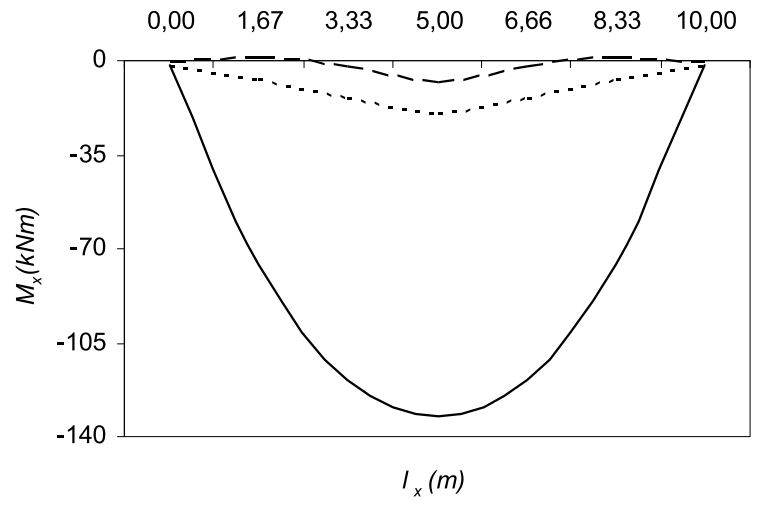

(b) Bending Moments for $\mathrm{H}=10 \mathrm{~m}, l_{y} / l_{x}=1.0$

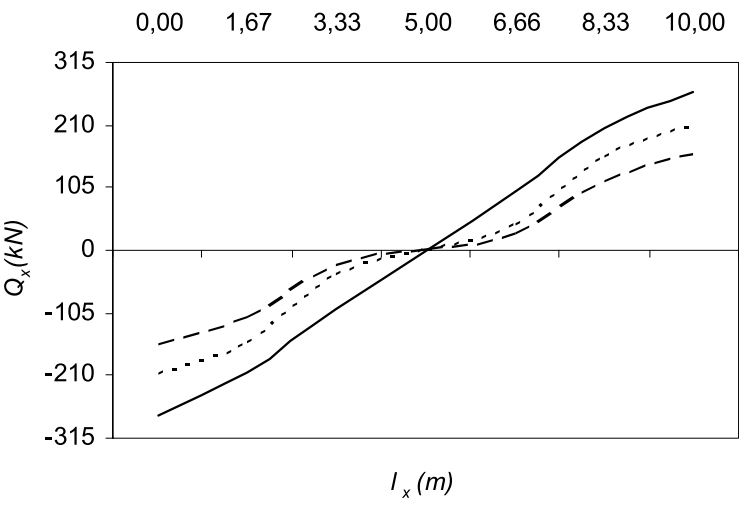

(c) Shear Forces for $\mathrm{H}=10 \mathrm{~m}, l_{y} / l_{x}=1.0$

Fig. 9. The variation of the maximum displacement, bending moments and shear forces. -, without beam; $\cdots, 1.00 \times 1.00$ beam;,$- 1.00 \times$ 1.50 beam.

decrease as the thickness of the beam increases. This behavior is understandable in that a plate with a beam or with larger thickness of the beam becomes more rigid.

The effect of the beam on the maximum displacement and on bending moments is considerable. And it can be said that the beams have stronger influence on the behavior of the plate than the aspect ratio.

\section{Conclusions}

In this study, 8-node (PBQ8) Mindlin plate element and Hughes element based on Timoshenko beam theory are adopted for the dynamic analysis of thick plates including beams on elastic foundations subjected to uniformly distributed loads. The effects of the thickness of the beam, the aspect ratio of the plate $\left(l_{y} / l_{x}\right)$ and the subsoil depth $(H)$ on the maximum displacement, bending moments and shear forces of thick plates on elastic foundations are investigated. The following conclusions drawn from the results obtained.

- The maximum displacements and bending moments increase with increasing subsoil depth $(H)$ up to a certain depth of the subsoil. Then, subsoil depth doesn't affect the results much.

- The maximum displacement and bending moment increase with increasing aspect ratio $\left(l_{y} / l_{x}\right)$.

- The maximum displacements, bending moments and shear forces decreases with increasing the thickness of the beam.

- The variation of displacement, bending moments and shear forces are changed significantly by adding the beams. 
- The effect of the subsoil depth on the behavior of the plate is generally larger than that of other parameters up to a certain depth of the subsoil while the effect of the beam on plate behavior is always significant.

\section{Appendix A}

The element mass matrix for 8-node Mindlin plate element (PBQ8) is given into sub matrices of $6 \times 6$ as follows

$$
\left[M_{p}\right]=a b\left[\begin{array}{c:c:c:c}
M_{1} & M_{2} & M_{3} & M_{4} \\
\hdashline M_{2} & M_{1} & M_{4} & M_{3} \\
\hdashline M_{3} & M_{4} & M_{5} & M_{6} \\
\hdashline M_{4} & M_{3} & M_{6} & M_{5}
\end{array}\right]
$$

where $a$ and $b$ are half-length of PBQ8 element in the $x$ and $y$ direction respectively and sub matrices,

$$
\begin{aligned}
& M_{1}=\frac{2}{15}\left[\begin{array}{c:c:c:c:c:c}
m_{1} & 0 & 0 & \frac{m_{1}}{3} & 0 & 0 \\
\hdashline 0 & m_{2} & 0 & 0 & \frac{m_{2}}{3} & 0 \\
\hdashline 0 & 0 & m_{3} & 0 & 0 & \frac{3}{3} \\
\hdashline 0 & 0 & 0 & m_{1} & 0 & 0 \\
\hdashline 0 & 0 & 0 & 0 & m_{2} & 0 \\
\hdashline 0 & 0 & 0 & 0 & 0 & m_{3}
\end{array}\right] \\
& M_{2}=\frac{1}{15}\left[\begin{array}{c:c:c:c:c:c}
m_{1} & 0 & 0 & \frac{2 m_{1}}{3} & 0 & 0 \\
\hdashline 0 & m_{2} & 0 & 0 & \frac{2 m_{2}}{3} & 0 \\
\hdashline 0 & 0 & m_{3} & 0 & 0 & \frac{2 m_{3}}{3} \\
\hdashline \frac{2 m_{1}}{3} & 0 & 0 & m_{1} & 0 & 0 \\
\hdashline 0 & \frac{2 m_{2}}{3} & 0 & 0 & m_{2} & 0 \\
\hdashline 0 & 0 & \frac{2 m_{3}}{3} & 0 & 0 & m_{3}
\end{array}\right] \\
& M_{3}=-\frac{2}{15}\left[\begin{array}{c:c:c:c:c:c}
m_{1} & 0 & 0 & \frac{4 m_{1}}{3} & 0 & 0 \\
\hdashline 0 & m_{2} & 0 & 0 & \frac{4 m_{2}}{3} & 0 \\
\hdashline 0 & 0 & m_{3} & 0 & 0 & \frac{4 m_{3}}{3} \\
\hdashline m_{1} & 0 & 0 & m_{1} & 0 & 0 \\
\hdashline 0 & m_{2} & 0 & 0 & m_{2} & 0 \\
\hdashline 0 & 0 & m_{3} & 0 & 0 & m_{3}
\end{array}\right] M_{4}=-\frac{2}{15}\left[\begin{array}{cc:c:c:c:c}
\frac{4 m_{1}}{3} & 0 & 0 & m_{1} & 0 & 0 \\
\hdashline 0 & \frac{4 m_{2}}{3} & 0 & 0 & m_{2} & 0 \\
\hdashline 0 & 0 & \frac{4 m_{3}}{3} & 0 & 0 & m_{3} \\
\hdashline \frac{4 m_{1}}{3} & 0 & 0 & \frac{4 m_{1}}{3} & 0 & 0 \\
\hdashline 0 & \frac{4 m_{2}}{3} & 0 & 0 & \frac{4 m_{2}}{3} & 0 \\
\hdashline 0 & 0 & \frac{4 m_{3}}{3} & 0 & 0 & \frac{4 m_{3}}{3}
\end{array}\right] \\
& M_{5}=\frac{4}{9}\left[\begin{array}{c:c:c:c:c:c}
\frac{8 m_{1}}{5} & 0 & 0 & m_{1} & 0 & 0 \\
\hdashline 0 & \frac{8 m_{2}}{5} & 0 & 0 & m_{2} & 0 \\
\hdashline 0 & 0 & \frac{8 m_{3}}{5} & 0 & 0 & m_{3} \\
\hdashline 0 & 0 & 0 & \frac{8 m_{1}}{5} & 0 & 0 \\
\hdashline 0 & 0 & 0 & 0 & \frac{8 m_{2}}{5} & 0 \\
\hdashline 0 & 0 & 0 & 0 & 0 & \frac{8 m_{3}}{5}
\end{array}\right] \quad M_{6}=\frac{4}{9}\left[\begin{array}{cc:c:c:c:c:c}
\frac{4 m_{1}}{5} & 0 & 0 & m_{1} & 0 & 0 \\
\hdashline 0 & \frac{4 m_{2}}{5} & 0 & 0 & m_{2} & 0 \\
\hdashline 0 & 0 & \frac{4 m_{3}}{5} & 0 & 0 & m_{3} \\
\hdashline m_{1} & 0 & 0 & \frac{4 m_{1}}{5} & 0 & 0 \\
\hdashline 0 & m_{2} & 0 & 0 & \frac{4 m_{2}}{5} & 0 \\
\hdashline 0 & 0 & m_{3} & 0 & 0 & \frac{4 m_{3}}{5}
\end{array}\right]
\end{aligned}
$$

where $m_{1}=\rho_{p} t_{p}+\frac{1}{3} \rho_{s} H$ and $m_{2}=m_{3}=\frac{1}{12} \rho_{p} t_{p}^{3}$.

\section{Appendix B}

The element mass matrix for 2-node Hughes element is given as follows 


$$
\left[M_{b}\right]=\left[\begin{array}{cccc}
\frac{m_{2} l}{3} & \frac{m_{2}}{2} & \frac{m_{2} l}{6} & -\frac{m_{2}}{2} \\
\frac{m_{2}}{2} & \frac{m_{1}+m_{2}}{l} & \frac{m_{2}}{2} & -\frac{m_{1}+m_{2}}{l} \\
\frac{m_{2} l}{6} & \frac{m_{2}}{2} & \frac{m_{2} l}{3} & -\frac{m_{2}}{2} \\
-\frac{m_{2}}{2} & -\frac{m_{1}+m_{2}}{l} & -\frac{m_{2}}{2} & \frac{m_{1}+m_{2}}{l}
\end{array}\right]
$$

where $l$ is length of Hughes element, $m_{1}=\rho_{b} t_{b}+\frac{1}{3} \rho_{s} H$ and $m_{2}=\frac{1}{12} \rho_{b} t_{b}^{3}$

\section{References}

[1] A.P.S. Selvaduari, Elastic Analysis of Soil-Foundation Interaction, Elsevier Scientific Publishing Company, Amsterdam, 1979.

[2] A. Labuschange, N.F.J. van Rensburg and A.J. van der Merwe, Vibration of a Reissner-Mindlin-Timoshenko plate-beam system, mathematical and computer modelling 50(7-8) (2009), 1033-1044.

[3] E.J. Sapountzakis and J.T. Katsikadelis, A new model for slab and beam structures-comparison with other models, Computers and Structures 80 (2002), 459-470.

[4] H.S. Shen, J. Ynag and L. Zhang, Free and forced vibration of reissner-mindlin plates with free edges resting on elastic foundations, Journal of Sound and Vibration 244(2) (2001), 299-320.

[5] E.J. Sapountzakis and V.G. Mokos, An improved model for the dynamic analysis of plates stiffened by parallel beams, Engineering Structures 30 (2008), 1720-1733.

[6] D.J. Gorman, Free vibration analysis of corner supported rectangular plates with symmetrically distributed edge beams, Journal of Sound and Vibration 263 (2003), 979-1003.

[7] N.F.J. Van Rensburg, L. Zietsman and A.J. Van Der Merwe, Solvability a Reissner-Mindlin-Timpshenko plate-beam vibration model, Journal of Applied Mathematics 74 (2009), 149-162.

[8] L. Yu, H.S. Shen and X.P. Huo, Dynamic responses of Reissner-Mindlin plates with free edges resting on tensionless elastic foundations, Journal of Sound and Vibration 299 (2007), 212-228.

[9] P.H. Wen and M.H. Aliabadi, Boundary element formulations for mindlin plate on an elastic foundation with dynamic load, Engineering Analysis with Boundary Elements 33 (2009), 1161-1170.

[10] K. Ozgan and A.T. Daloglu, Effect of transverse shear strain on plates resting on elastic foundation using modified vlasov model, Thin Walled Structures 46(2008), 1236-1250.

[11] K. Ozgan and A.T. Daloglu, Application of the modified vlasov model to the free vibration analysis of thick plates resting on elastic foundation, Shock and Vibration 16 (2009), 439-454.

[12] A. Turhan, A Consistent Vlasov Model for Analysis of Plates on Elastic Foundations Using the Finite Element Method, Ph. D. Thesis, The Graduate School of Texas Tech. University. Lubbock, Texas, 1992.

[13] K. Ozgan, Finite Element Analysis of Thick Plates on Elastic Foundation Using Modified Vlasov Model and Determination of The Effective Depth of Soil Stratum, Ph. D. Thesis, Karadeniz Technical University, Trabzon, Turkey, 2007 (in Turkish).

[14] K.J. Bathe, Finite Element Procedures, Upper Saddle River, NJ: Prentice-Hall, 1996.

[15] W. Weaver and P.R. Johnston, Finite Elements for Structural Analysis, Englewood Cliffs, NJ: Prentice-Hall, Inc., 1984.

[16] V. Kolar and I Nemec, Modelling of Soil Structures Interactions, Praha, Academia, 1989.

[17] A. Daloglu, A. Dogangun and Y. Ayvaz, Dynamic Analysis of foundation plates using a consistent vlasov model, Journal of Sound and Vibration 224(5) (1999), 941-951.

[18] A.T. Daloglu and K. Ozgan, The effective depth of soil stratum for plates resting on elastic foundation, Structural Engineering and Mechanics 18(2) (2004), 263-276. 

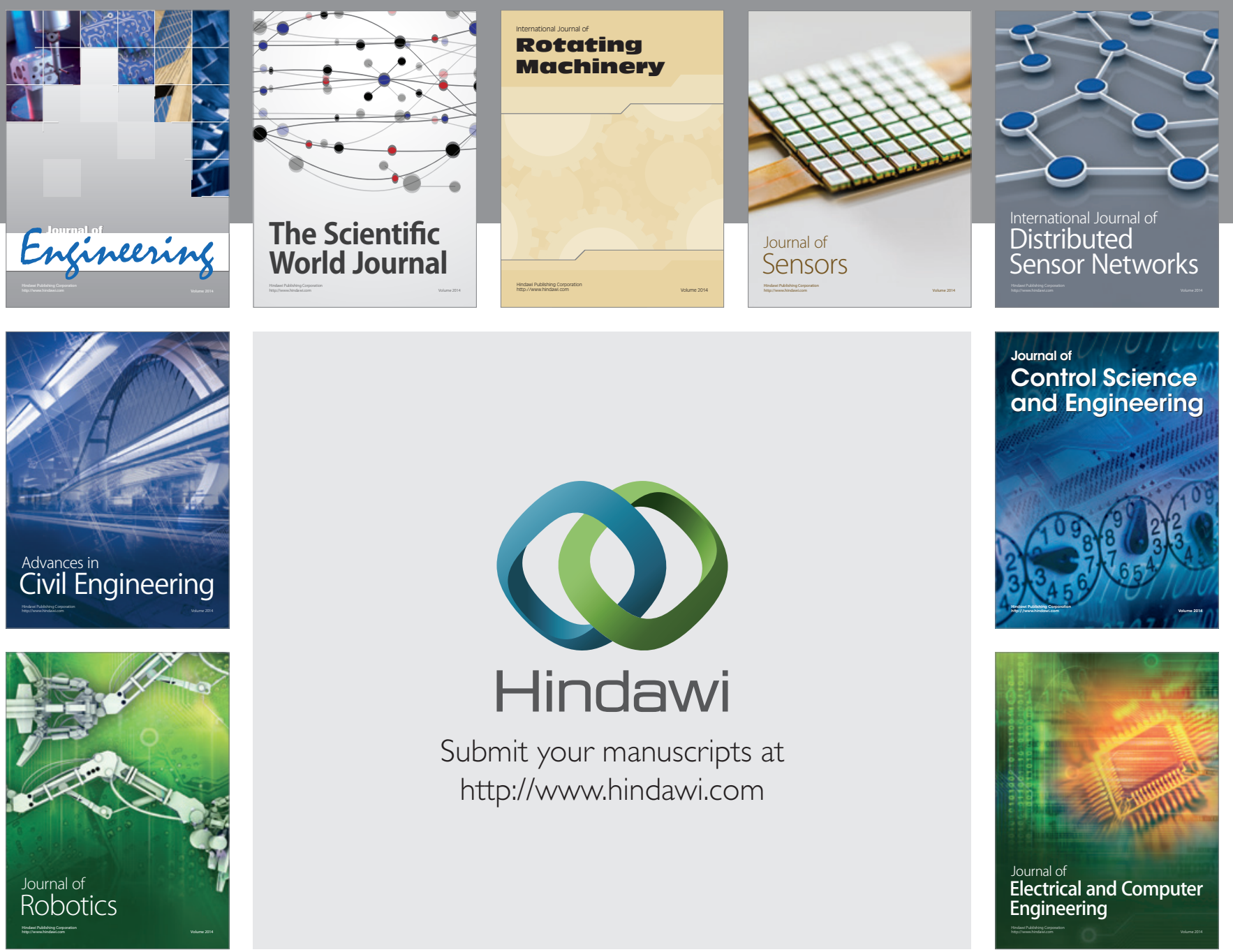

Submit your manuscripts at

http://www.hindawi.com
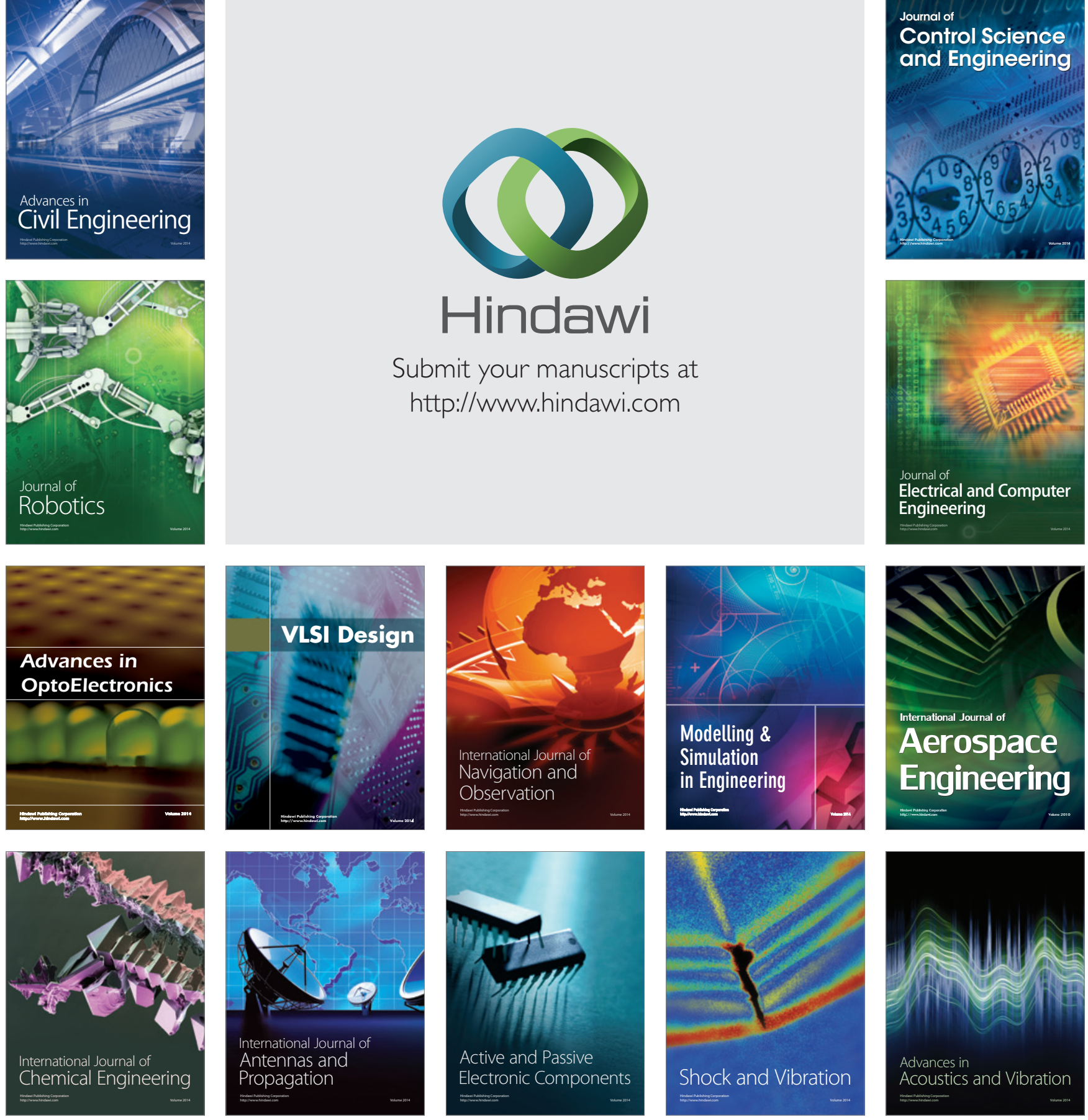